\title{
A New Design Approach and Framework for Elderly Care Robots
}

\section{Adam Poulsen}

School of Computing \& Mathematics

Charles Sturt University

Bathurst, New South Wales

Email: apoulso2@postoffice.csu.edu.au

\section{Oliver K. Burmeister}

School of Computing \& Mathematics

Charles Sturt University

Bathurst, New South Wales

Email: oburmeister@csu.edu.au

\section{David Tien}

School of Computing \& Mathematics

Charles Sturt University

Bathurst, New South Wales

Email: dtien@csu.edu.au

\begin{abstract}
A relatively new area within information systems is the design of robotic healthcare. This narrative review considers the question, how does one ethically design an elderly care robot? To answer this question, robot ethicists consider the ethical impact of robots, how designers ought to design robots ethically, and how a robot design ought to be, so its behaviour is ethical. The latter consideration defines another field of study, machine ethics. Machine ethicists ask, how does one design a robot information system to behave ethically? Thus, robot ethics is concerned with the ethics of design practice, whereas machine ethics is concerned with the ethics of the product designed. The findings from this narrative review point the way forward to how one can answer both questions with a new design approach that is grounded in care and professional ethics, value sensitive design, and the integration of two machine ethics schools of thought.
\end{abstract}

Keywords Ethics, Robot, Care, Systems, DSR. 


\section{Introduction}

The question of how to conduct ethical design practice is not new, however, it is when applied to elderly healthcare robotics. In attempting to answer the robot ethics question, robot ethicists present care robot design approaches. Human-computer interaction (HCI) has long been an important area of information systems (IS) research relating to healthcare. For example, a proven healthcare IS approach, user-centred design (UCD) (Burmeister et al. 2016; Burmeister et al. 2015; Hagedorn et al. 2016; Pakrasi et al. 2015; Schnall et al. 2016), and another, value sensitive design (VSD) (Burmeister 2010; Burmeister 2016; Burmeister and Kreps 2018; Friedman 1996; Friedman et al. 2006; Teipel et al. 2016), have both been used and adapted to guide care robot design. Human-robot interaction (HRI) is relatively new in IS healthcare research. Care centered value sensitive design (CCVSD) (van Wynsberghe 2013a) is one of those adaptions of traditional approaches that accounts for HRIs. It is an approach that outlines the design theories, concepts, processes, methods, and evaluation criteria for ethically creating a care robot design. As shown in the continued literature review below, both UCD and VSD have been used in care robot design.

Recently machine ethicists have focussed on determining what a robot's behaviour ought to be-within the design process. As yet this is not done well and one of the contributions of this study is to help address this important consideration in IS research. Machine ethicists have tried to answer this question by contributing design frameworks to literature. A framework consists of a guiding school of thought, a set of ethics, an ethics-delivery method for providing a machine with ethics or how to turn ethics into behaviours, and an exemplary design. The following sections review the literature, beginning with robot ethics and related research. Next, the new approach suggested by the literature review is described.

\section{Literature Review}

The methodology for this study followed that of the narrative literature review beginning with a wide search that yielded 80 articles and then refining the results further to 31. It has been suggested that a narrative review of even five studies with a wide range of methods on a topic could draw sound conclusions because narrative literature reviews differ from empirical reviews of a large number of studies (Baumeister and Leary 1997). First, they can answer broad questions by identifying patterns and making connections across a number of studies on a topic with differing methodologies. Second, they can develop and validate conclusions and theories after the findings of individual empirical studies have been published. Third, they can accept questions not being answered by the current data and can instead highlight and offer ways to address underlying problems. Fourth, they can make different conclusions from the data, namely that the hypothesis was correct, could be correct but not proven, may or may not be correct, or is proven wrong. Finally, using a number of studies on a topic with differing methodological approaches can identify flaws, raise doubts in past conclusions and offer alternatives for any particular method.

Although narrative reviews have been criticised for being vulnerable to author bias, it has also been suggested that such criticism could be addressed by using a systematic approach to establish rigorous and evidence-based conclusions for both qualitative and quantitative reviews (Green et al. 2006). As such, this study applied an objective and scientific approach for selecting and reviewing the literature and included components generally expected by different guidelines for reporting studies, such as, Consolidated Standards of Reporting Trials (CONSORT), STrengthening the Reporting of OBservational studies in Epidemiology (STROBE), Preferred Reporting Items for Systematic Reviews and MetaAnalyses (PRISMA), and Standards for Reporting Diagnostic accuracy studies (STARD) (International Committee of Medical Journal Editors 2016).

To locate literature specifically about robotic healthcare, an advanced search criterion was employed. Using Charles Sturt University's Primo search engine, the following 4 strings were searched: "care robot", "care robots", "care robot design", and "care robot ethics". The potential results of each of the 4 searches were limited to only include journal articles published during or after 2005. Books, reviews, reports, and video presentations, as well as anything published before 2005, were excluded. The results were sorted by relevance. The first 2 pages of each of the 4 searches, each having 10 articles, were used. This presented a total of 80 articles published since 2005. Each abstract of those 80 articles was read to determine if the paper presented either an approach or a framework. Thirty-one articles were found to fit that brief, 9 approach related articles and 22 framework related articles. Of the 9 approach related articles, 6 were selected to highlight the use of traditional approaches, as well as adapted approaches, in care robot design. Moreover, of the 22 framework related articles, 11 were selected as exemplary representations for the purposes of this paper. 


\subsection{Robot \& Machine Ethics}

Robot ethics is concerned with the ethical impact of robots, how designers ought to design robots ethically, and how a robot design ought to behave (Malle 2016). The latter consideration is machine ethics, the study of how robots ought to act (Wallach and Allen 2009). A clear distinction between robot and machine ethics can be made in relation to a shared concern-design. Robot ethics explores the ethics of the designer while undertaking the design process. Robot ethics research considers how a roboticist goes about designing a robot ethically (a design approach). By contrast, machine ethics explores the ethics of the robot. Machine ethics research considers how a robot is to act ethically, how the design of the robot ought to be so that it is ethical, and how to provide those ethics (a design framework). A legal perspective provides a clear distinction between the two. In exploring the regulation of robot design and robot behaviour design, Leenes and Lucivero (2014) identify the legal distinction between the regulation of human beings (robot ethics) and the regulation of robot behaviour through design (machine ethics).

Design approaches describe how a designer ought to undergo the practice of design. Each approach provides practical design practices for one or more of the following for the design process: data gathering, data analysis, discussion of data, and application of data to the design process. UCD is an approach which brings users into the design process (Duarte and Guerra 2012; Vredenburg et al. 2002); by doing so it hopes to create products which are focussed on bettering the user experience (Abras 2004; Strömberg 2005). VSD, another approach, aims to produce systems which are sensitive to indirect and direct stakeholders' values by involving them in the design process (Friedman et al. 2006). UCD has been used for designing socially assistive long-term care robots (Di Nuovo et al. 2018), elderly care service robot interactions (Mast et al. 2012), and recommendations for every day, domestic robots (Sung 2011). VSD has also been applied to care robot design. It has been used for designing seizure detection systems (van Andel et al. 2015), retrospective analysis of therapeutic care robots (Melson et al. 2005), and humanoid care robot design (Cheon and $\mathrm{Su}$ 2016). These are examples of attempting to answer the robot ethics question. CCVSD is another attempt. CCVSD is a care robot specific approach that incorporates care ethics and Tronto's (1993) care values as moral elements of a care practice as normative criteria for evaluating a care practice (van Wynsberghe 2013b).

Two machine ethics schools of thought can be identified. Moral robotics defines robots which are capable of making moral decisions (Wallach and Allen 2009). Moreover, moral robots are explicit (or full) ethical agents in the sense that they have ethical principles explicitly represented in their system for them to use to make moral decisions (Moor 2006). The antithesis of an explicitly ethical moral robot is an implicit ethical agent, belonging to good robotics, which has ethics embedded into its strictly preprogramed implicit behaviour (Moor 2006). Machine ethicists present moral and good robot frameworks to try to answer the machine ethics question. A design framework encompasses the following components involved in designing the ethical behaviour of a care robot.

1. School - The machine ethics school of thought followed. The school is either moral robotics where the author recommends a design which has explicit ethical decision-making, or good robotics where the author recommends a design which is implicitly ethical.

2. Ethics set - A set of the ethics which ought to be provided to a care robot.

3. Ethics-delivery method - A method for translating the ethics into ethical behaviours.

4. Exemplary design - An exemplary care robot design.

Moral robotics frameworks are easy to derive from literature. Machine ethicists following the moral robotics school are attempting to provide a care robot with explicit ethical behaviours, thus the set of ethics and ethics-delivery method are explicitly stated. Good robotics frameworks, on the other hand, aren't as clear; this is inherent in designing implicit ethical agents. Implicit agents, or good robots, are designed to be ethical, not to make ethical decisions. Thus, a good robot framework doesn't strictly require a method, although one may be derived if the designer describes the technical details. However, they certainly feature a set of ethics that a designer either intentionally or unintentionally followed when making design decisions. Table 1 shows exemplary frameworks from both schools.

\begin{tabular}{|c|c|c|c|c|}
\hline Author(s) & School & Ethics set & Ethics-delivery method & Exemplary design \\
\hline
\end{tabular}


Shaw et al. Moral Relative and evolving (2018) robotics ethics. Human-like transparency to allow the care robot to justify its decisions. Imperfect ethics like a human.

(Anderson

and

Anderson

2008; 2017)

Moral robotics

Rossian deontology. Deontology declares universal moral duties and considers actions to be either moral or immoral based on the act itself rather than the consequence (Alexander and Moore 2016). Duties can collide and thus W.D. Ross suggested that the actual duty to be followed was that which was most stringent or 'prima facie' ('first face') based on an individual's

obligation.

Sharkey and Good Deontology ethics Sharkey

(2011;

(2012a;

2012b);

Sparrow

(2002;

Sparrow and

Sparrow

(2006)

robotics
Meacham Good Care ethics and Studley robotics (2017)

\section{A New Design Approach for Elderly Care Robots}

Bottom-up learning method to provide ethics. Evolution of ethics that allows the care robot to learn new ethics in practice. A group-dynamic decision-making principle.

A hybrid method made up of bottom-up casebased learning and a top-down principle that controls the learning process. The ethical training cases are predetermined, by an ethicist, to represent good or bad ethical decisions. Thus, the ethical principle that is learnt is inferred by the decisions of the ethicist; what is learnt is not random, it is predetermined.
An action-driven method in which ethics are implicitly coded into care robot actions. The authors hold action to be the most important thing in robot delivered care, not robotic moral competence, agency, or any other element of moral robotics.
A care robot design which learns ethics subjectively without top-down limitations. The design includes a 'robot brain' with many 'voices' that test each other to reach a consensus.

A care robot design that is controlled by Rossian deontological prima facie duties, but the way it chooses which duty is right in situ is taught to the care robot explicitly and with predetermined results.

Collectively, the authors suggest a design that acts implicitly ethically, programmed with behaviours judged to be good by deontological standards. Contrasting the last framework, this one features no ethical decisionmaking. The authors promote respecting patient autonomy and dignity as key ethical duties.

A care robot design that is implicitly ethical in its actions which are determined by a designer determining what 'meaningful context' is. 


\subsection{Good Care}

IS research has shown the importance of values in systems design thinking in many areas, including online applications (Chesney et al. 2009), in areas of e-Health (Boonstra and Van Offenbeek 2010), and emerging technologies (Dainow 2017). IS in healthcare is no exception. Good human-delivered care is grounded in both care ethics (Beauchamp and Childress 2009; Pakrasi et al. 2015) and professional ethics (Bernoth et al. 2016; Bowern et al. 2006; Burmeister 2017); robot-delivered care should be no different. Care ethics describes good care decisions as being "determinative in practice" (Beauchamp 2004). They come from a caregiver's subjective interpretation of a patient's unique needs, which determines how we ought to behave to ensure those needs are met. Just as a human carer must make decisions affecting those in their care, during the practice of caring (Upton 2011), so too care robots must do so at run-time. In care ethics, it is considered ethical if a moral decision arises from the 'good' which is internal to practice, rather than external normative moral criteria or principles (Vallor 2011). Care ethics present principles that describe moral behaviour and action when taking care of someone (Vanlaere and Gastmans 2011), or patient care values that should be respected. Professional ethics, made up of codes of ethics, set ethical principles, values, practices, and processes for caregivers to uphold. Principles and processes do not determine the act which is best in specific situations. Instead, carers make this determination in practice, and care robots should do the same. However, that said, there are some normative principles within professional ethics that are intrinsic to care and must be respected by industry or organisation regulation. For example, the safety of a patient is regulated by professional codes of ethics and it is not up to carers to decide not to ensure intrinsic safety; however, how to achieve it is to be determined in practice. Thus, care ethics are determined by a carer's interpretation of a patient's individual needs as observed in the carer-patient relationship. Moreover, professional ethics are determined objectively by other parties. The European parliament's report on robot legislation (Commission on Civil Law Rules on Robotics 2017), International Organisations of Standardization's standard for care robots (International Organization for Standardization 2014), and International Council of Nurses' code of ethics (2012) all describe professional ethics required in robotdelivered care.

Care values describe the principles and behaviours which recipients of care expect from social interactions. Due to its non-normative grounding in the importance of the subjective context, care ethics is best for describing the extrinsic care values in care practices. By contrast, due to its normative grounding in the importance of objective safety and a basic level of care, professional ethics best describes the intrinsic values of care practices. Extrinsic values are those that help to achieve intrinsic ones (Zimmerman 2015). Extrinsic care values are a patient's unique wants and desires within a care practice, whereas intrinsic care values are those which describe the end goal of the care practice. Van Wynsberghe (2013a) claims that it is through the manifestation of values, during the design process of care robots, that one comes to understand what care really is in practice. Thus, care values are a good basis for design as they simply express patient needs, can be transformed into coded behaviours, and are supported by literature on technology design.

\subsection{Previous Frameworks Integrating Moral and Good Robotics Design Considerations}

Madl and Franklin (2015) suggest that a care robot be provided with a decision-making mechanism that is constrained to the domain and functionalities where the robot was intended to function, consists of a combination of explicit rules and implicit, heuristic strategies when processing decisions, and is adaptive. Constraining the robot's ability to act and giving it implicitly ethical decision processes is making it implicitly ethical, or a good robot. Moreover, allowing it to make decisions from explicit rules and allowing it to adapt its decision-making is making it explicitly ethical, or a moral robot.

Madl and Franklin (2015) do not provide a design approach for how to design for such considerations, nor do they make a clear distinction between extrinsic and intrinsic values; while the authors' framework answers the machine question by integrating moral and good robotics, it doesn't answer the robot ethics question. A framework that does make such a distinction has been presented by Poulsen and Burmeister (2018). The authors offer 'the attentive framework', an elderly care robot design framework that recommends a moral robot design which is governed by dynamic extrinsic values to provide care ethics, as well as unchanging intrinsic values to provide professional ethics (although they do not identify the latter, nor connect them to the schools). The validity of the framework has been proven in a heuristic expert evaluation and an international online survey. Participants were presented with an exemplary design created using the framework, a medicine delivery robot that made dynamic value trade-offs in run-time. That is, it was a moral robot that adapts its behaviour to extrinsic patient values but always respected intrinsic values. The care robot presented to participants had an ethical decision-making 
process that hypothetically allows it to interpret patient care values, structure extrinsic ones into a list, set value priorities in that list depending on how much the patient desired them (as interpreted by the robot), reaffirmed the priority consistently, and adapted its behaviour based on the ordering of extrinsic values to customise care to each patient. As for intrinsic values, those were said to be off-limits to the robot, thus it couldn't ever decide which intrinsic value was more important than another nor let an extrinsic value consideration be held higher than an intrinsic one. Upon testing the framework and design as a hypothetical artefact, 3 of the 4 experts and $76 \%$ of the survey participants thought the care robot was sensitive in making dynamic, extrinsic value decisions. The full results and further discussion are published elsewhere (Poulsen 2018; Poulsen et al. 2018a; Poulsen et al. 2018b). The study presented a framework and therefore attempted to answer the machine ethics question, which it did in theory. However, the authors never answered the robot ethics question, they did not provide an approach to practically designing a care robot based on the framework. Furthermore, what is missing from that work is a clear identification and integration of moral and good robotics and a concerted effort to merge robot and machine ethics. Therefore, this proposed research presents a new design approach to do just that. One that accounts for ethical design practice, the integration of the schools, and a merging of robot and machine ethics while attempting to design a care robot outlined by that framework.

The literature review has indicated several challenges with current approaches and frameworks. First, approaches fail to provide guidance in designing the ethical behaviour of a care robot (machine ethics design concerns). Second, frameworks are unhelpful in guiding design practice (robot ethics design concerns). Third, approaches and frameworks do not demonstrate good care as is described here. The literature review also suggests how to overcome these problems-an innovatively combined approachframework (referred to simply as the new design approach). In the new approach, the non-contradictory, advantageous design elements of the machine ethics schools of thought are merged to support the creation of a CCVSD-adapted, implicit-explicit approach. The approach declares that robotic behaviour ought to be ethical by making them an implicitly and explicitly ethical agent so that the care provided is implicitly and explicitly good. That is, both questions can be answered with an approach that accounts for the design concerns of both robot and machine ethics together, rather than as exclusive design considerations. The new approach also accounts for good care through its grounding in care and professional ethics.

\section{A New Approach is Needed}

The new approach suggested by the above literature review needs to be grounded in good care determinants and needs to integrate the good robotics and moral robotics schools. This innovative design approach, which is merged with a framework, would inherently integrate robot and machine ethics with the shared concern for design-this integration answers the robot and machine ethics questions at the same time. The focus on design means that the design approach must present a comprehensive and guiding design approach and an interweaved framework suited to the approach. The design focus also distinguishes it from previous integration attempts that are centrally concerned with regulation and ethics. The design approach could be adapted from CCVSD where values are dependent on specific care practices, however with a small difference. To be grounded in good care determinants, it should take patient care values, and value interpretations, to be constantly changing. Moreover, the approach should recognise that each care practice has values that are unique to each patient and that those values change as patients age, are exposed to new experiences, or have interactions with new technologies (Sharkey 2014; van Wynsberghe 2013a). To integrate the two schools, the robot design itself should also be able to adapt to changing care values. Thus, accounting for individual interpretations of values, each patient's different value priorities, differences in value types, and how each patient's values may change is central to the new design approach-values in motion design (VMD).

To undertake VMD, first, a VSD conceptual investigation should commence. Conceptually, a designer should establish a model of user requirements and an initial framework adapted from the attentive framework. Second, a VSD empirical-technical investigation should start and participants for a design workshop should be recruited. A design workshop describes a focus group that brings in elderly persons, elderly caregivers, and a researcher to serve as a guide to conduct the VMD design process. In the design workshop, participants should be presented with the model of user needs and the initial framework which they will use to produce a care robot design. If possible, an ethicist and a robotics or HRI expert should be brought in as design workshop participants to help from their unique perspectives. However, if such participants cannot be sourced, the researcher may serve their roles. Participatory design such as this is a well-founded method in design practice (Duarte and Guerra 2012; Vredenburg et al. 2002).

From a care ethics perspective, VMD could promote and support the provision of subjective, dynamic care. From a professional ethics perspective, caregivers must uphold codes of ethics to ensure a standard 
of quality care. From a VSD perspective, care ethics decisions could be described as the respecting of extrinsic patient care values whereas professional ethics decisions can be described as ensuring that intrinsic patient care values are provided. Putting extrinsic and intrinsic care values to use in the design approach's design process should be done with the value tool. VMD would be more focused on IS design considerations than physical ones, thus the value tool and framework emphasise software and robot behaviour design. To guide a practitioner undertaking the design process outlined by the approach, they would be provided with the value tool to help distinguish between extrinsic and intrinsic values, as well as a framework to provide design guidelines on how to translate values into a care robot design.

\subsection{The Value Tool}

Previously it was mentioned that values are dependent on care practices, a concept supported by literature (Tronto 1993; van Wynsberghe 2013a). Designers undertaking VMD would need a method to unravel a care practice to reveal all the values, distinguish between extrinsic and intrinsic values, and design a care robot to account for each value correctly according to its type. The value tool might serve as that method. The value tool consists of a table and steps for using the table. The steps have been adapted from the CCVSD methodology which explains how to extract values from a care practice by elaborating on it in a human-delivered care scenario, and then in a robot-delivered care scenario. With those two elaborations in competing contexts, the practitioner can compare them to reveal the positive and negative effects on care values due to the care robot introduction. The tool is to be used first by experts to establish a care robot design which satisfies all intrinsic values, as well as some recommendations for extrinsic values. Then it is to be used by design workshop participants to identify any further extrinsic values and amend the design to account for them. Using the value tool during the initial expert design stage, as well as for the design workshop, is to be carried out as follows.

\section{Expert steps}

1. Select a care practice and elaborate it, in two competing contexts, to reveal all relevant values. List it in the value tool table.

2. Identify which values are intrinsic and extrinsic in the care practice. List them in the value tool table.

a. To identify which values are intrinsic, experts are to consult regulation, nursing codes of conduct, and healthcare standards. Moreover, experts can provide insight into what is required in terms of patient safety and basic care in the care practice under consideration.

b. To identify which values are extrinsic, experts should simply to consider all values which aren't intrinsic as extrinsic.

3. Design for professional ethics care decisions by taking intrinsic values and discussing what end goals are needed to ensure intrinsic values, then consider hardcoded robot components to ensure those goals are always achieved. Use the value tool table to structure that discussion and list the design elements. Consult the framework to see how that can be achieved.

4. Design for care ethics decisions by taking extrinsic values and discussing how the robot could adapt its behaviour to respect each extrinsic value while achieving its intrinsic value goals, then design dynamic robot components for it to do so in practice. Use the value tool table to structure that discussion and list the design elements. Consult the framework to see how that can be achieved.

\section{Workshop participant steps}

1. Examine the care robot designed by experts.

2. Select a care practice listed in the value tool table and elaborate it, in two competing contexts, to reveal all relevant extrinsic values only. Elaboration might consist of having a walkthrough of the care practice with multiple participants, as well as discussing it as a group.

3. Identify which values are extrinsic in the care practice. List them in the value tool table.

a. To identify which values are extrinsic, elderly persons and caregivers who are participating could be consulted. The elderly persons should be encouraged to discuss what they desire from a caregiver who is performing a care practice. What their desires are is the extrinsic values of that care practice. 
4. Design for care ethics decisions by taking extrinsic values (those identified by both the experts and the participants) and discussing how the robot could adapt its behaviour to respect each extrinsic value while achieving its intrinsic value goals, then design dynamic robot components for it to do so in practice. Use the Table 2 value tool to structure that discussion and list the design elements. Consult the framework to see how that can be achieved.

\begin{tabular}{l|ll|ll}
\hline $\begin{array}{l}\text { Care } \\
\text { practice }\end{array}$ & $\begin{array}{l}\text { Intrinsic } \\
\text { values }\end{array}$ & $\begin{array}{l}\text { Robot design elements needed to } \\
\text { ensure intrinsic values }\end{array}$ & $\begin{array}{l}\text { Extrinsic } \\
\text { values }\end{array}$ & $\begin{array}{l}\text { Robot design elements needed } \\
\text { to ensure extrinsic values }\end{array}$ \\
\hline
\end{tabular}

Table 2. The value tool table

\subsubsection{Example of Using the Value Tool}

Take the care practice 'bath patient' for example. Without VMD, designers may program a robot to close the curtains around the bath to respect the patient's dignity. However, what if the patient feels threatened by that, or they feel alone with a robot they do not entirely understand or trust, or they do not like enclosed spaces, or they feel like they are endangered because they are hidden from a human caregiver seeing them if they were drowning, or perhaps they would like to talk to their partner in the doorway. Instead what if intrinsic and extrinsic values were declared. One could examine nursing codes of ethics to reveal the intrinsic values of bathing a patient: hygiene and safety for example. Then if caregivers and patients are consulted, extrinsic values could be discovered: privacy, dignity, social contact, emergency help, happiness, touch, eye contact, independence, and comfort for example. The intrinsic values should be embedded into the design of the care robot, the assurance of them shouldn't be conflicted by any other possible action. In this case, to ensure the value of hygiene the robot could have a cleaning function to clean the patient, have cameras to see any dirt on the patient, and arms and hands to hold a sponge. To ensure safety, the cameras might observe the patient to ensure their head is above water and to see any distress and one arm could be at the ready to grab the patient under the arm to pull them up if they are drowning. Now that hygiene and safety have been implicitly embedded into the design, one can consider the extrinsic values. Such values should be represented explicitly in the robot's physical and information systems. One can design any number of functions/behaviours (that can even conflict with each other) and provide them to the IS for it to determine during run-time which is the best behaviour to suit the patient's desires. For privacy, the robot could avert its anthropomorphic eyes and could delete the video logs (a few hours after the bath just in case the patient is hurt, and the recordings are needed for liability. The robot ought to be able to sense when an injury occurs to account for this). For dignity, the robot could shut a curtain around the bath. For happiness, the robot could turn on the radio or TV or talk to the patient. Do this for all extrinsic values. Such behaviours may conflict because the care robot is to observe patients and decide which behaviour they would prefer during runtime-what the attentive framework presented above called dynamic value trade-offs in run-time. This feature is also recommended by VMD since it merges with the attentive framework.

\begin{tabular}{l|ll|ll}
\hline $\begin{array}{l}\text { Care } \\
\text { practice }\end{array}$ & $\begin{array}{l}\text { Intrinsic } \\
\text { values }\end{array}$ & $\begin{array}{l}\text { Robot design elements needed to } \\
\text { ensure intrinsic values }\end{array}$ & $\begin{array}{l}\text { Extrinsic } \\
\text { values }\end{array}$ & $\begin{array}{l}\text { Robot design elements needed } \\
\text { to ensure extrinsic values }\end{array}$ \\
$\begin{array}{l}\text { Bath } \\
\text { patient }\end{array}$ & Hygiene & $\begin{array}{l}\text { A cleaning function to clean the } \\
\text { patient. Cameras to see any dirt } \\
\text { on the patient. Arms and hands } \\
\text { to hold a sponge. }\end{array}$ & Privacy & $\begin{array}{l}\text { Avert anthropomorphic eyes. } \\
\text { Delete video recordings (a few } \\
\text { hours after the bath just in } \\
\text { case the patient is hurt, and } \\
\text { the recordings are needed for } \\
\text { liability). }\end{array}$ \\
Shut a curtain around the \\
bath.
\end{tabular}

Table 3. An example use of the value tool table

\section{IS Systems Thinking is Required}

Following the design-science research (DSR) methodology is a proven best practice for IS design research (Helms et al. 2010; Hevner et al. 2004; Peffers et al. 2007). DSR is described by Hevner et al. 
(2004) as being both a set of activities (process) and a design product (artefact). It focuses on developing innovative or more efficient and effective ways to address unresolved problems (Hevner et al. 2004). DSR research must be relevant and rigorous. That is, it must have a clear contribution to literature and that one must construct that contribution (a design artefact) with iterative build-evaluate phases (Hevner et al. 2004). DSR might prove a good research methodology for testing VMD. In keeping with the DSR methodology, VMD could be empirically tested to determine its practicality. Furthermore, it could be evaluated for its effectiveness and efficacy compared to existing approaches, or what DSR refers to as methods (Hevner et al. 2004). Through testing VMD, a new care robot design, or what DSR labels an instantiation (Hevner et al. 2004), would emerge. The instantiation would consist of physical robotic components and an IS. This could also be evaluated against criteria for meeting user needs, or what DSR refers to as a model (Hevner et al. 2004). Following DSR processes, based on an evaluation of the method and the instantiation, the method could be amended from an analysis of that evaluation to make it more effective and efficient for practitioner use, as well as to make sure it best meets user needs as described by the model. In this way, DSR could be used to produce an effective and efficient approach which accounts for good care and addresses the robot and machine ethics questions.

\section{Conclusion}

The narrative review points to how VMD design could be an all-in-one solution to the robot and machine ethics questions. VMD is an instructive approach grounded in good care and CCVSD for guiding design practice (robot ethics). It is supplemented by the recommended framework which is also grounded in good care (machine ethics). With VMD, designers could ethically produce a care robot design that demonstrates good care through the provision of implicitly safe and good professional ethics decisions (good robotics), as well as explicitly customised care ethics decisions (moral robotics). DSR could be used to empirically test the efficiency and effectiveness of VMD in practice to bring it out of theory and into the hands of practitioners. Although this paper has focussed on care for elderly persons, there is no reason why VMD could not be used in care for others such as the handicapped, soldiers suffering from post-traumatic stress disorder, or cancer patients weakened by chemotherapy.

\section{References}

Abras, C., Maloney-Krichmar, D., Preece, J. 2004. "User-Centered Design," in: Encyclopedia of HumanComputer Interaction, W. Bainbridge (ed.). Thousand Oaks: Sage Publications.

Alexander, L., and Moore, M. 2016. "Deontological Ethics," in: The Stanford Encyclopedia of Philosophy, E.N. Zalta (ed.). Stanford, CA: Stanford University.

Anderson, M., and Anderson, S. L. 2008. "Ethel: Toward a Principled Ethical Eldercare System," AAAI Fall Symposium: AI in Eldercare: New Solutions to Old Problems.

Anderson, M., and Anderson, S. L. 2017. "A Value Driven Agent: Instantiation of a Case-Supported Principle-Based Behavior Paradigm," in: AAAI 2016 Workshop on AI, Ethics \& Society.

Baumeister, R. F., and Leary, M. R. 1997. "Writing Narrative Literature Reviews," Review of General Psychology (1:3), p. 311.

Beauchamp, T. L. 2004. "Does Ethical Theory Have a Future in Bioethics?," The Journal of Law, Medicine \& Ethics (32:2), pp. 209-217.

Beauchamp, T. L., and Childress, J. F. 2009. Principles of Biomedical Ethics. Oxford: Oxford University Press.

Bernoth, M., Burmeister, O. K., Morrison, M., Islam, M. Z., Onslow, F., and Cleary, M. 2016. "The Impact of a Participatory Care Model on Work Satisfaction of Care Workers and the Functionality, Connectedness and Mental Health of Community-Dwelling Older People," Issues in Mental Health Nursing (37:6), pp. 1-7.

Boonstra, A., and Van Offenbeek, M. 2010. "Towards Consistent Modes of E-Health Implementation: Structurational Analysis of a Telecare Programme's Limited Success," Information Systems Journal (20:6), pp. 537-561.

Bowern, M., Burmeister, O. K., Gotterbarn, D., and Weckert, J. 2006. "Ict Integrity: Bringing the Acs Code of Ethics up to Date," Australasian Journal of Information Systems (13:2), pp. 168-181.

Burmeister, O. K. 2010. "Websites for Seniors: Cognitive Accessibility," International Journal of Emerging Technologies and Society (8:2), pp. 99-113.

Burmeister, O. K. 2016. "The Development of Assistive Dementia Technology That Accounts for the Values of Those Affected by Its Use," Ethics and Information Technology (18:3), pp. 185-198.

Burmeister, O. K. 2017. "Professional Ethics in the Information Age," Journal of Information, Communication \& Ethics in Society (15:4), pp. 348-356. 
Burmeister, O. K., Bernoth, M., Dietsch, E., and Cleary, M. 2016. "Enhancing Connectedness through Peer Training for Community-Dwelling Older People: A Person Centred Approach," Issues in Mental Health Nursing (37:6), pp. 1-6.

Burmeister, O. K., Islam, M. Z., Dayhew, M., and Crichton, M. 2015. "Enhancing Client Welfare through Better Communication of Private Mental Health Data between Rural Service Providers," Australasian Journal of Information Systems (19), pp. 1-14.

Burmeister, O. K., and Kreps, D. 2018. "Power Influences Upon Technology Design for Age-Related Cognitive Decline Using the Vsd Framework," Ethics and Information Technology (20:3).

Cheon, E., and Su, N. M. 2016. "Integrating Roboticist Values into a Value Sensitive Design Framework for Humanoid Robots," in: The Eleventh ACM/IEEE International Conference on Human Robot Interaction. Christchurch, New Zealand: IEEE Press, pp. 375-382.

Chesney, T., Coyne, I., Logan, B., and Madden, N. 2009. "Griefing in Virtual Worlds: Causes, Casualties and Coping Strategies," Information Systems Journal (19:6), pp. 525-548.

Commission on Civil Law Rules on Robotics. 2017. "P8_Ta(2017)oo51."

Dainow, B. O. 2017. "Threats to Autonomy from Emerging Icts," Australasian Journal of Information Systems (21), pp. 1-16.

Di Nuovo, A., Broz, F., Wang, N., Belpaeme, T., Cangelosi, A., Jones, R., Esposito, R., Cavallo, F., and Dario, P. 2018. "The Multi-Modal Interface of Robot-Era Multi-Robot Services Tailored for the Elderly," Intelligent Service Robotics (11:1), pp. 109-126.

Duarte, J., and Guerra, A. 2012. "User-Centered Healthcare Design," Procedia Computer Science (14), pp. 189-197.

Friedman, B. 1996. "Value-Sensitive Design," Interactions (3:6), pp. 17-23.

Friedman, B., Kahn, P. H. J., and Borning, A. 2006. "Value Sensitive Design and Information Systems," in Human-Computer Interaction and Management Information Systems: Foundations, P. Zhang and D. Galletta (eds.). New York: M. E. Sharpe, pp. 348-372.

Green, B. N., Johnson, C. D., and Adams, A. 2006. "Writing Narrative Literature Reviews for PeerReviewed Journals: Secrets of the Trade," Journal of Chiropractic Medicine (5:3), pp. 101-117.

Hagedorn, T. J., Krishnamurty, S., and Grosse, I. R. 2016. "An Information Model to Support UserCentered Design of Medical Devices," Journal of Biomedical Informatics (62), pp. 181-194.

Helms, R., Giovacchini, E., Teigland, R., and Kohler, T. 2010. "A Design Research Approach to Developing User Innovation Workshops in Second Life," 2010 (3:1).

Hevner, A. R., March, S. T., Park, J., and Ram, S. 2004. "Design Science in Information Systems Research," MIS Q. (28:1), pp. 75-105.

International Committee of Medical Journal Editors. 2016. "Preparing for Submission." from http://www.icmje.org/recommendations/browse/manuscript-preparation/preparing-forsubmission.html

International Council of Nurses. 2012. "The ICN Code of Ethics for Nurses." from http://www.icn.ch/who-we-are/code-of-ethics-for-nurses/

International Organization for Standardization. 2014. "Robots and Robotic Devices -- Safety Requirements for Personal Care Robots." from https://www.iso.org/standard/53820.html

Leenes, R., and Lucivero, F. 2014. "Laws on Robots, Laws by Robots, Laws in Robots: Regulating Robot Behaviour by Design," Law, Innovation and Technology (6:2), pp. 193-220.

Madl, T., and Franklin, S. 2015. "Constrained Incrementalist Moral Decision Making for a Biologically Inspired Cognitive Architecture," in A Construction Manual for Robots' Ethical Systems: Requirements, Methods, Implementations, R. Trappl (ed.). Cham: Springer International Publishing, pp. 137-153.

Malle, B. F. 2016. "Integrating Robot Ethics and Machine Morality: The Study and Design of Moral Competence in Robots," Ethics and Information Technology (18:4), pp. 243-256.

Mast, M., Burmester, M., Krüger, K., Fatikow, S., Arbeiter, G., Graf, B., Kronreif, G., Pigini, L., Facal, D., and Qiu, R. 2012. "User-Centered Design of a Dynamic-Autonomy Remote Interaction Concept for Manipulation-Capable Robots to Assist Elderly People in the Home," J. Hum.-Robot Interact. (1:1), pp. 96-118.

Meacham, D., and Studley, M. 2017. "Could a Robot Care? It's All in the Movement," in Robot Ethics 2.o: From Autonomous Cars to Artificial Intelligence, P. Lin, K. Abney and R. Jenkins (eds.). Oxford University Press.

Melson, G. F., Peter H. Kahn, J., Beck, A. M., Friedman, B., Roberts, T., and Garrett, E. 2005. "Robots as Dogs?: Children's Interactions with the Robotic Dog Aibo and a Live Australian Shepherd," in: CHI 'O5 Extended Abstracts on Human Factors in Computing Systems. Portland, OR, USA: ACM, pp. 1649-1652.

Moor, J. H. 2006. "The Nature, Importance, and Difficulty of Machine Ethics," IEEE Intelligent Systems (21:4), pp. 18-21. 
Pakrasi, S., Burmeister, O. K., McCallum, T. J., Coppola, J. F., and Loeb, G. 2015. "Ethical Telehealth Design for Users with Dementia," Gerontechnology (13:4), pp. 383-387.

Peffers, K., Tuunanen, T., Rothenberger, M. A., and Chatterjee, S. 2007. "A Design Science Research Methodology for Information Systems Research," Journal of Management Information Systems (24:3), pp. 45-77.

Poulsen, A. 2018. "Dynamic Value Trade-Offs in Run-Time to Provide Good, Customised Patient Care with Robots." Charles Sturt University, p. 116.

Poulsen, A., and Burmeister, O. K. 2018. "Overcoming Carer Shortages with Care Robots: Dynamic Value Trade-Offs in Run-Time," Australasian Journal of Information Systems (22).

Poulsen, A., Burmeister, O. K., and Kreps, D. 2018a. "The Ethics of Inherent Trust in Care Robots for the Elderly," in: This Changes Everything - ICT and Climate Change: What Can We Do?, D. Kreps, C. Ess, L. Leenen and K. Kimppa (eds.). Poznan, Poland: Springer International Publishing, pp. 314-328.

Poulsen, A., Burmeister, O. K., and Tien, D. 2018b. "Care Robot Transparency Isn't Enough for Trust," in: 2018 IEEE Region 10 Symposium (TENSYMP). Sydney, Australia.

Schnall, R., Rojas, M., Bakken, S., Brown, W., Carballo-Dieguez, A., Carry, M., Gelaude, D., Mosley, J. P., and Travers, J. 2016. "A User-Centered Model for Designing Consumer Mobile Health (Mhealth) Applications (Apps)," Journal of Biomedical Informatics (60), pp. 243-251.

Sharkey, A. 2014. "Robots and Human Dignity: A Consideration of the Effects of Robot Care on the Dignity of Older People," Ethics and Information Technology (16:1), pp. 63-75.

Sharkey, A., and Sharkey, N. 2011. "Children, the Elderly, and Interactive Robots," Robotics \& Automation Magazine, IEEE (18:1), pp. 32-38.

Sharkey, A., and Sharkey, N. 2012a. "The Eldercare Factory," Gerontology (58:3), pp. 282-288.

Sharkey, A., and Sharkey, N. 2012b. "Granny and the Robots: Ethical Issues in Robot Care for the Elderly," Ethics and Information Technology (14:1), pp. 27-40.

Shaw, N. P., Stockel, A., Orr, R. W., Lidbetter, T. F., and Cohen, R. 2018. "Towards Provably Moral Ai Agents in Bottom-up Learning Frameworks," in: AAAI/ACM Conference on Artificial Intelligence, Ethics, and Society. New Orleans, USA.

Sparrow, R. 2002. "The March of the Robot Dogs," Ethics and Information Technology (4:4), pp. 305318.

Sparrow, R., and Sparrow, L. 2006. "In the Hands of Machines? The Future of Aged Care," Minds and Machines (16:2), pp. 141-161.

Strömberg, H., Leikas, J., Ikonen, V., Iivari, N., Jokela, T., \& Leurs, N. 2005. "User-Centred Design Guidelines for Methods and Tools," Nomadic Media.

Sung, J. Y. 2011. "Towards the Human-Centered Design of Everyday Robots," in: College of Computing. Georgia Institute of Technology.

Teipel, S., Babiloni, C., Hoey, J., Kaye, J., Kirste, T., and Burmeister, O. K. 2016. "Information and Communication Technology Solutions for Outdoor Navigation in Dementia," Alzheimer's \& Dementia: The Journal of the Alzheimer's Association (12:6), pp. 695-707.

Tronto, J. C. 1993. Moral Boundaries a Political Argument for an Ethic of Care. Abingdon, United Kingdom: Routledge.

Upton, H. 2011. "Moral Theory and Theorizing in Health Care Ethics," Ethical Theory and Moral Practice (14:4), pp. 431-443.

Vallor, S. 2011. "Carebots and Caregivers: Sustaining the Ethical Ideal of Care in the Twenty-First Century," Philosophy \& Technology (24:3), pp. 251-268.

van Andel, J., Leijten, F., van Delden, H., and van Thiel, G. 2015. "What Makes a Good Home-Based Nocturnal Seizure Detector? A Value Sensitive Design," PLOS ONE (10:4), p. e0121446.

van Wynsberghe, A. 2013a. "Designing Robots for Care: Care Centered Value-Sensitive Design," Science and Engineering Ethics (19:2), pp. 407-433

van Wynsberghe, A. 2013b. "A Method for Integrating Ethics into the Design of Robots," Industrial Robot: An International Journal (40:5), pp. 433-440.

Vanlaere, L., and Gastmans, C. 2011. "A Personalist Approach to Care Ethics," Nursing Ethics (18:2), pp. 161-173.

Vredenburg, K., Mao, J.-Y., Smith, P. W., and Carey, T. 2002. "A Survey of User-Centered Design Practice," in: Proceedings of the SIGCHI Conference on Human Factors in Computing Systems. Minneapolis, Minnesota, USA: ACM, pp. 471-478.

Wallach, W., and Allen, C. 2009. Moral Machines: Teaching Robots Right from Wrong. New York: Oxford University Press.

Zimmerman, M. J. 2015. "Intrinsic Vs. Extrinsic Value," in: The Stanford Encyclopedia of Philosophy, E.N. Zalta (ed.). Stanford, CA: Stanford University. 
Copyright: (c) 2018 Adam Poulsen, Oliver K. Burmeister, and David Tien. This is an open-access article distributed under the terms of the Creative Commons Attribution-NonCommercial 3.0 Australia License, which permits non-commercial use, distribution, and reproduction in any medium, provided the original author and ACIS are credited. 